\title{
Network Pharmacology-Based Investigation For Predicting Active Ingredients And Potential Targets Of Strychnos Nux-Vomica L. In Treating Multiple Myeloma
}

\section{Yan Zhou}

Zhejiang University of Traditional Chinese Medicine: Zhejiang Chinese Medical University Jianping Shen

Zhejiang University of Traditional Chinese Medicine First Affiliated Hospital: Zhejiang Hospital of Traditional Chinese Medicine

\section{Keting Jin}

Zhejiang University of Traditional Chinese Medicine: Zhejiang Chinese Medical University

Chenjun Lin

Zhejiang University of Traditional Chinese Medicine: Zhejiang Chinese Medical University

\section{Zirui Hong}

Zhejiang University of Traditional Chinese Medicine: Zhejiang Chinese Medical University

Shu Deng ( $\square$ dengshudmn@163.com )

Zhejiang University of Traditional Chinese Medicine First Affiliated Hospital: Zhejiang Hospital of

Traditional Chinese Medicine https://orcid.org/0000-0001-8353-6705

\section{Research}

Keywords: Strychnos nux-vomica L., Network pharmacology, Multiple Myeloma

Posted Date: October 30th, 2020

DOI: https://doi.org/10.21203/rs.3.rs-97494/v1

License: (c) (i) This work is licensed under a Creative Commons Attribution 4.0 International License. Read Full License 


\section{Abstract}

Background: Strychnos nux-vomica L. (SN)『a classic Chinese herb, have long been used for the treatment of cancer for many years, However, the pharmacological mechanisms of SN in treatment of Multiple myeloma L.remain vague.The aim of this study was to examine the network pharmacological potential effects of SN on Multiple myeloma using a systems pharmacology approach.

Methods: we collected putative targets of SN based on the Traditional Chinese Medicine System Pharmacology database区and oral bioavailability and drug-likeness was screened using absorption, distribution, metabolism, and excretion (ADME) criteria. the network of the interactions among the putative targets of SN and known therapeutic targets of Multiple myeloma was built by using the STITCH database. Then, topological parameters, "Degree” ,"Closeness” and"Betweenness" were calculated to identify the hub targets in the network. Furthermore, the hub targets were imported to the Database for Annotation, Visualization and Integrated Discovery to perform a pathway enrichment analysis.

Results: 60 of the identified potential targets of the SN were also Multiple Myeloma- related targets, including 14 putative targets of SN were observed to be major hubs in terms of topological importance.Additionally,the results of pathway enrichment analysis indicated that targets of $\mathrm{SN}$ in treating Multiple Myeloma were mainly clustered into multiple biological processes by activating on several signaling pathways(PI3K-Akt, p38-MAPK, Ras/Raf/MEK/ERK pathways), which implied that these were involved in the underlying mechanisms of SN on Multiple Myeloma.

Conclusions: Our works successfully explain the potential effects of SN for Multiple Myeloma treatment via the molecular mechanisms predicted by network pharmacology.Moreover,our present outcomes might shed light on the further clinical application of SN in treating Multiple Myeloma.

\section{Background}

Multiple myeloma (MM) is a clonal plasma cell hematologic malignancy that accounts for approximately more than $10 \%$ of all hematologic cancers. Advances in therapy over the last 2 decades have prolonged

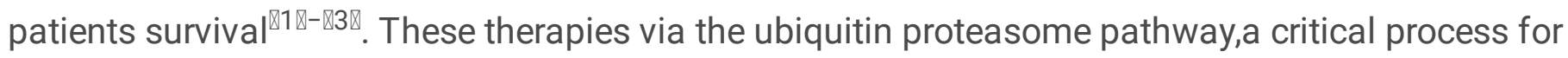
plasma cell survival to degradate target protein ${ }^{\bigotimes 4 \rrbracket}$.Examples includes proteasome inhibitors (such as bortezomib) and the immunomodulatory drugs (such as lenalidomide and Thalidomide). However, these drugs have yielded desired responses while have also contribute to certain adverse reactions such as peripheral neuropathy $\llbracket$ damage to liver and kidney function. Therefore,it is urgent to discover potential therapeutic targets and find novel and safe treatment strategies.

The use of traditional Chinese medicine (TCM) has obtained more and more acceptance all over the world due to its therapeutic efficacy and fewer side effects ${ }^{858-87 区}$. Different from Western Medicine,TCM

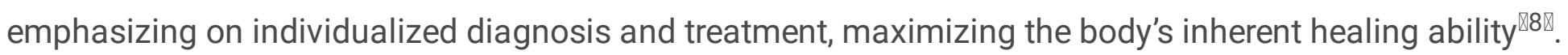


It's characterized by multiple ingredients while make it hard to distinguish the active ingredients and to identify the potential targets of the chemicals.

Strychnos nux-vomica L. (SN) belongs to the genus Strychnos of the family Loganiaceae, mainly contains alkaloids with the fuctions of anti-tumor,anti-inflammatory effects and effects on the nervous system,etc. In traditional Chinese medicine, SN have long been used for the treatment of liver cancer Q98- $812 \rrbracket$, The latest research also found SN can dramatically inhibit the growth of U266B1 and RPMI 8226

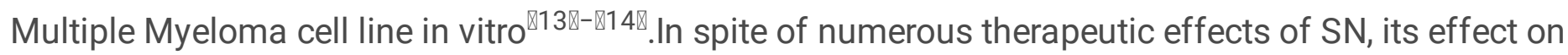
hematologic malignancies is still ill-defined. Besides,similar to other TCM formulas, SN is a multicomponent and multi-target agent, it is hard to investigate the pharmacological mechanisms. With the rapid progress of bioinformatics, systems biology, and poly-pharmacology, network pharmacology based approaches have been proven to be a powerful way to decipher the complex mechanisms of action of

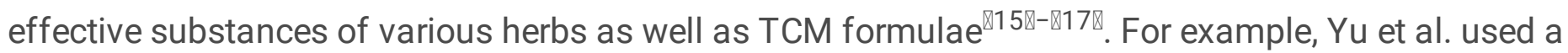
network pharmacology strategy to explore pharmacological mechanisms of Zuojinwan for treatment of gastritis ${ }^{\mathbb{1} 8}$. He et al.also predicting active ingredients and potential targets of LiuWei DiHuang Pill in treating type 2 diabetes mellitus by the use of network pharmacology ${ }^{819 \rrbracket}$.

In this research, we exert a pharmacology network and experimental verification combination method to investigate how SN exerts the therapeutic effects on MM.The fowchart of the experimental procedures of our study is shown in Fig. 1.

\section{Methods}

\section{Data preparation}

\section{Chemical ingredients database building and ADME Screening}

To collect the ingredients in SN, we performed a search by Traditional Chinese Medicine Systems

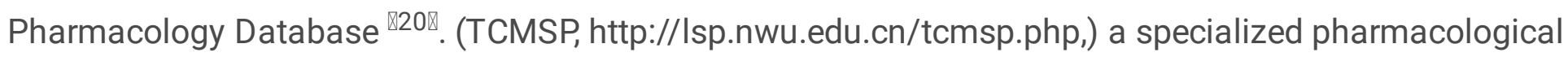
platform for TCM. A total of 500 Chinese herbal medicines and 30069 ingredients from the Chinese Pharmacopoeia (2010 edition) were registered ${ }^{\mathbb{2} 21 \rrbracket}$.After that,we used absorption, distribution, metabolism, and excretion (ADME) to select bioactive components that contribute to its therapeutic effects.Based on previous studies,we regard the candidate components with Oral bioavailability $(\mathrm{OB}) \geq 30 \%$,D rug-likeness $(\mathrm{DL}) \geq 0.18$ as pharmacologically active ${ }^{\llbracket 20 \rrbracket, 822 \rrbracket}$.

\section{The prediction of targets acting on Multiple Myeloma}

We collected Multiple myeloma targets from GeneCards (https://www.genecards.org/, ver.4.9.0) database,which is an online catalog of human genes and genetic diseases that combines detailed drug data with target genes information ${ }^{\text {223 }}$. 


\section{Target genes related to the identified compounds}

SN active ingredient targets gene was collected by TCMSP and PubChem database (https://pubchem.ncbi.nlm.nih.gov), we fished targets with a screening online tool called PharmMapper

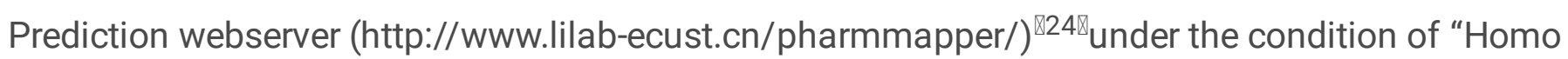
sapiens" species setting. Gene information including name, gene ID and organism was confirmed using

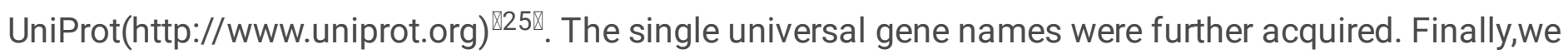
matched the retrieved SN active ingredient targets to the known MM-related targets by the STRING (https://string-db.org/, ver. 11.0) database to draw the data of protein-protein Interactions (PPI) ${ }^{\mathbb{2} 6 \mathbb{Z}}$.

\section{Network Construction And Analysis}

The compound-target (C-T) network $\square$ Strychnos nux-vomica L. target-MM target interactional (T-T)network and the target pathway(T-P)network were all visualized by using Cytoscape ver. 3.5.1

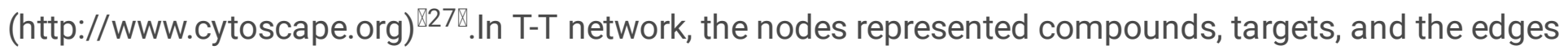
displayed the interactions between two nodes. Moreover, we selected "Degree" $₫$ "Closeness" and "Betweenness" to evaluate the topological features of every node in the interaction network and

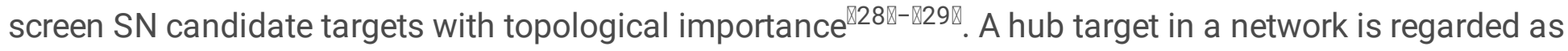
a crucial node and used to measure the essence of the whole network.Based on previous study, a node was determined to be a hub if its "Degree" and "Betweenness" were greater than or equal to twice the median values while the "Closeness" were greater than median values of the network ${ }^{\otimes 30 \rrbracket-831 \rrbracket}$.

\section{Functional Enrichment Analysis}

We used DAVID (https://david.ncifcrf.gov/v6.8,2019.8.14) datebase for the T-P network, which includes GO-biological process (BP) and KEGG (http://www.kegg.jp/, 2019.8.14) enrichment analysis to further probe the vital biological process of achieved targets and to validate the reliability of the integrated results $^{\llbracket 32 \pi}$.

\section{Results}

\section{Active compounds in Strychnos nux-vomica L}

A total of 113 compounds were identified in SN, all identified compounds were subjected to ADME screening, and 13 of the 113 had $\mathrm{OB} \geq 30 \%$ and $\mathrm{DL} \geq 0.18$. The 13 active compounds that were obtained are listed in (Table 1).

To further understand the pharmacological mechanisms of SN,we constructed an compound-target network by linking the active compounds with their potential targets. There are totally 221 nodes and 390 edges composed the C-T network,including the 13 active compounds and 341 targets(Fig. 2). These active 
compounds interacting with multiple targets, participated in the regulation of multiple targets, which signify that SN act synergistically on these targets, exerted pharmacological effects in Multiple myeloma.

Table 1

The list of 13 compounds of Strychnos nux-vomica $L$. and their $\mathrm{OB}$ and $\mathrm{DL}$

\begin{tabular}{|c|c|c|c|c|c|}
\hline NO. & Medicine & MOLNAME & compound & $\mathrm{OB}(\%)$ & DL \\
\hline 1 & $\begin{array}{l}\text { Strychnos nux- } \\
\text { vomica L. }\end{array}$ & MOL001040 & $\begin{array}{l}\text { (2R)-5,7-dihydroxy-2-(4- } \\
\text { hydroxyphenyl)chroman-4-one }\end{array}$ & 42.36 & 0.21 \\
\hline 2 & $\begin{array}{l}\text { Strychnos nux- } \\
\text { vomica L. }\end{array}$ & MOL001476 & (S)-Stylopine & 51.15 & 0.85 \\
\hline 3 & $\begin{array}{l}\text { Strychnos nux- } \\
\text { vomica L. }\end{array}$ & MOL003410 & Ziziphin_qt & 66.95 & 0.62 \\
\hline 4 & $\begin{array}{l}\text { Strychnos nux- } \\
\text { vomica L. }\end{array}$ & MOL003411 & Icaride A & 48.74 & 0.43 \\
\hline 5 & $\begin{array}{l}\text { Strychnos nux- } \\
\text { vomica L. }\end{array}$ & MOL003413 & Isostrychnine $\mathrm{N}$-oxide (I) & 35.45 & 0.80 \\
\hline 6 & $\begin{array}{l}\text { Strychnos nux- } \\
\text { vomica L. }\end{array}$ & MOL003414 & Isostrychnine N-oxide (II) & 37.33 & 0.80 \\
\hline 7 & $\begin{array}{l}\text { Strychnos nux- } \\
\text { vomica L. }\end{array}$ & MOL003418 & Lokundjoside_qt & 32.82 & 0.76 \\
\hline 8 & $\begin{array}{l}\text { Strychnos nux- } \\
\text { vomica L. }\end{array}$ & MOL003432 & vomicine & 47.56 & 0.65 \\
\hline 9 & $\begin{array}{l}\text { Strychnos nux- } \\
\text { vomica L. }\end{array}$ & MOL003433 & brucine-N-oxide & 49.17 & 0.38 \\
\hline 10 & $\begin{array}{l}\text { Strychnos nux- } \\
\text { vomica L. }\end{array}$ & MOL003436 & Isobrucine & 33.58 & 0.80 \\
\hline 11 & $\begin{array}{l}\text { Strychnos nux- } \\
\text { vomica L. }\end{array}$ & MOL003440 & Brucine N-oxide & 52.63 & 0.38 \\
\hline 12 & $\begin{array}{l}\text { Strychnos nux- } \\
\text { vomica L. }\end{array}$ & MOL000449 & Stigmasterol & 43.83 & 0.76 \\
\hline 13 & $\begin{array}{l}\text { Strychnos nux- } \\
\text { vomica L. }\end{array}$ & MOL000492 & $(+)$-catechin & 54.83 & 0.24 \\
\hline
\end{tabular}

The target data on MM from Drugbank was finally collected 1350 targets which the Relevance score is over 2.54 as Multiple Myeloma-related target. Notably, 60 of the identified potential targets of the SN were also be proved as Multiple Myeloma-related targets by the Venn diagram(Fig. 3a).Subsequently, to further select the hub targets of SN in treating Multiple Myeloma, we imported compound-disease co-targets 
information into STRING datebase and set the upper limit of protein amount of "no more than 50 interactors". The intersection network consisting of 109 nodes and 1530 edges (Fig. 3b).The topological feature analysis of the PPI selected targets used "degree","Betweenness" and "Closeness" to determine hub targets and finally 14 targets were screened based on the values of topological parameters. The details are shown in ( Fig. 3c).

Nodes includes AKT1 (degree = 76), EGFR(degree = 69), ALB(degree = 65), MAPK1(degree = 64), EGF(degree $=64), \operatorname{VEGFA}($ degree $=64), \operatorname{CCND1}($ degree $=63), \operatorname{SRC}($ degree $=61)$ and JUN (degree $=61)$ have higher degrees, the number of edges of each node is large as well,which suggesting that they play important roles in the treatment of Multiple Myeloma (Fig. 4). The topological parameters are shown in (Table 2).

Table 2

Topological feature values of all the hub targets for $\mathrm{SN}$ in treatment of MM.

\begin{tabular}{|llll|}
\hline Node name & Degree & Closeness & Betweenness \\
\hline AKT1 & 76.00 & 92.00 & 660.44 \\
\hline EGFR & 69.00 & 88.33 & 507.28 \\
\hline ALB & 65.00 & 86.50 & 804.30 \\
\hline EGFK1 & 64.00 & 86.00 & 480.35 \\
\hline VEGFA & 64.00 & 85.83 & 338.45 \\
\hline CCND1 & 63.00 & 85.17 & 353.51 \\
\hline SRC & 61.00 & 84.33 & 375.28 \\
\hline JUN & 61.00 & 84.50 & 273.06 \\
\hline CASP3 & 59.00 & 83.33 & 171.71 \\
\hline HSP90AA1 & 58.00 & 82.83 & 611.79 \\
\hline ESR1 & 58.00 & 83.00 & 324.07 \\
\hline MAPK8 & 53.00 & 80.33 & 270.90 \\
\hline FN1 & 52.00 & 79.67 & 303.70 \\
\hline
\end{tabular}

\section{GO biological process and KEGG pathway enrichment analysis}

The biological processes were mainly involved in regulation of apoptotic apoptosis(G0:0043066), signal transduction(GO:0007165), regulation of transcription(G0:0045893) and cell proliferation (G0:0008284). 
(Fig. 5).Moreover, according to the p'values of enriched pathways and their correlation with MM, we were most interested in the following signaling pathwayss including PI3K-Akt,p38 MAPK and Ras/Raf/MEK/ERK pathway(Fig. 6 and Table 3).

Table 3

Representative enriched KEGG pathway of the hub targets of SN in treating MM.

\begin{tabular}{|lllll|}
\hline Pathway & $\begin{array}{l}\text { Gene } \\
\text { Count }\end{array}$ & P'Value & $\begin{array}{l}\text { Pathway } \\
\text { ID }\end{array}$ & Associated Genes \\
\hline $\begin{array}{l}\text { PI3K-Akt signaling } \\
\text { pathway }\end{array}$ & 8 & $\begin{array}{l}4.79 E- \\
07\end{array}$ & hsa04151 & $\begin{array}{l}\text { HSP90AA1, CCND1, EGF, FN1, MAPK1, } \\
\text { AKT1, EGFR, VEGFA }\end{array}$ \\
$\begin{array}{l}\text { MAPK signaling } \\
\text { pathway }\end{array}$ & 7 & $\begin{array}{l}1.79 E- \\
06\end{array}$ & hsa04010 & $\begin{array}{l}\text { JUN, MAPK8, EGF, CASP3, MAPK1, AKT1, } \\
\text { EGFR }\end{array}$ \\
\hline $\begin{array}{l}\text { Ras signaling } \\
\text { pathway }\end{array}$ & 6 & $\begin{array}{l}2.40 \mathrm{E}- \\
05\end{array}$ & hsa04014 & $\begin{array}{l}\text { MAPK8, EGF, MAPK1, AKT1, EGFR, } \\
\text { VEGFA }\end{array}$ \\
\hline
\end{tabular}

\section{Discussion}

Network pharmacology analysis of traditional medicines is becoming a popular approach for researching the treatment of diseases manifested by complex and diverse factors such as multiple myeloma. We explored the potential mechanism by the network pharmacology approach to investigate the complex of ingredients, unknown targets, and pharmacological mechanisms in herb. Although a few studies have confirmed SN with the fuctions of anti-tumor ${ }^{89 \mathbb{Z}}$,but the therapeutic effects of $\mathrm{SN}$ against multiple myeloma has not yet been clearly elucidated.In this study, a total of 13 potential active ingredients of SN on Multiple Myeloma were screened through a series of network pharmacological methods, which corresponded to 14 hub targets by a topological method.Previous studies have reported the association between these targets and Multiple Myeloma.For instance, AKT10EGFR HSP90AA1 and ESR1 served as

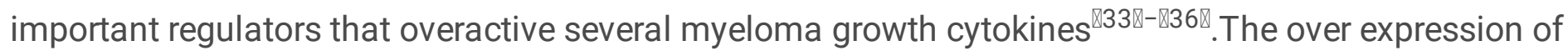
MAPK8 and MAPK1 allows the p38 MAPK signaling pathway activated and inhibits osteoblast

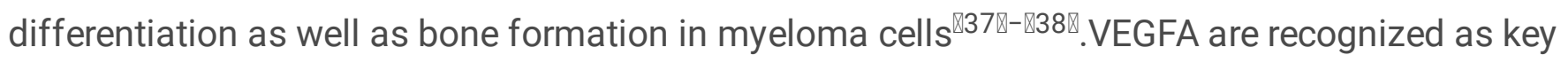
angiogenic factors in Multiple Myeloma,which stimulates vascular permeability, EC migration,

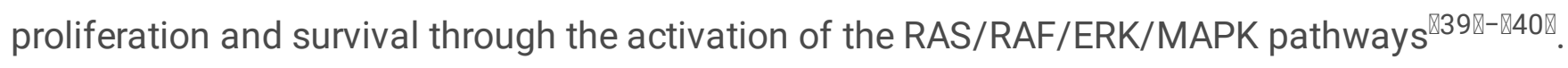

Enrichment analysis of GO-BP and KEGG on the hub targets further suggests that SN could exerts the therapeutic effects on Multiple Myeloma multiple biological processes by activating on several signaling pathways.As a result, we found that the targets have relatively large number of connections with the PI3K-Akt signaling pathway,MAPK signaling pathway and Ras signaling pathway.PI3K-Akt signaling pathway has been improved plays an integral role in MM disease biology,which is activated as a result of loss of function of tumor suppressor genes. Akt is a nodal regulator of cellular survival pathways, the activation of the Akt promote cell proliferation, anti-apoptosis, and metabolism signals leading to myeloma cells growth and survival ${ }^{841 ه-843 \rrbracket}$. We demonstrate that p38 MAPK signaling pathway constitutively activated in human myeloma, which has been implicated in inhibiting osteoblast 


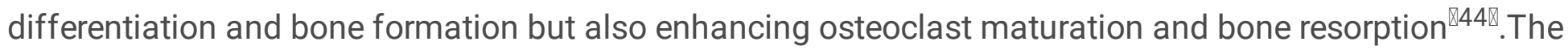
Ras/Raf/MEK/ERK pathway is critical for the proliferation of myeloma cells,implicated in the angiogenesis in multiple myeloma by the driven of vascular endothelial growth factor (VEGF) 8458-847区 There are also diverse signaling pathways enhanced the expression of several growth factors and cytokines thus promite the accumulate clonal malignant plasma cells in the bone marrow ${ }^{8480}$.

\section{Conclusion}

Based on the results of this study, we investigate the potential mechanism of SN against multiple myeloma.13 bioactive compounds of $\mathrm{SN}$ and 60 targets associated with multiple myeloma were selected. Furthermore,14 core targets including AKT1, EGFR, MAPK1 MAPK8 might be the hub targets for $\mathrm{SN}$ on multiple myeloma treatment.Despite the valuable discoveries, Further in vitro and in vivo experimental validation is needed to support our research.

\section{List Of Abbreviations}

SN: Strychnos nux-vomica L.;MMIMultiple myeloma ; TCM: Traditional Chinese Medicine;ADME: Absorption, distribution, metabolism, and Excretion; OB: oral bioavailability; DL:drug-likeness; GO: Gene Ontology; KEGG: Kyoto Encyclopedia of Genes and Genomes; PPI: protein-protein interaction; DAVID: Database for Annotation, Visualization and Integrated Discovery; C-T: compound-target; T-T: target-target; T-P: targetpathway;AKT1:RAC-alpha serine/threonine-protein kinase;EGFR: Epidermal growth factor receptor;HSP90AA1: Heat shock protein 90 alpha family class A member 1;ESR1:Estrogen receptor;MAPK: Mitogen-activated protein kinase;VEGF:Vascular endothelial growth factor.

\section{Declarations}

\section{Ethics approval and consent to participate}

Not applicable.

\section{Consent for publication}

Not applicable.

\section{Availability of data and materials}

The datasets used/or analysed during the current study are available from the corresponding author on reasonable request.

\section{Competing interest}

The authors declare that they have no conflicts of interest regarding the publication of this paper. 


\section{Funding}

This article was funded by the Construction Project of Zhejiang basic public welfare research program(2021ZB092).This funding source had no role in the design, execution, analyses, interpretation of the data, or decision to submit results.

\section{Authors' contributions}

YZ and KTJ collected data, performed the analyses, conducted the literature search, and Contributed to the writing of the manuscript. SD and JPS took part in the design of the study and made a revision of the manuscript. CJL and YRH conceptualised and designed the study, coordinated the study, helped interpret findings, and critically reviewed the manuscript. All authors read and approved the final manuscript.

\section{Acknowledgments}

Not applicable.

\section{References}

1. Kumar SK, Rajkumar SV, Dispenzieri A, et al. Improved survival in multiple myeloma and the impact of novel therapies. Blood. 2008;111(5):2516-20.

2. Kumar SK, Dispenzieri A, Lacy MQ, et al. Continued improvement in survival in multiple myeloma: changes in early mortality and outcomes in older patients. Leukemia. 2014;28(5):1122-8.

3. Gonsalves WI. Rajkumar SV,Go RS,et al.Trends in survival of patients with primary plasma cell leukemia: a. population-based analysisBlood. 2014;124(6):907-12.

4. Caro JL, Davies FE. PIKing the next therapeutic target in multiple myeloma. Haematologica. 2020;105(6):1474-5.

5. Li B, Xu X, Xia W, et al. A systems biology approach to understanding the mechanisms of action of Chinese herbs for treatment of cardiovascular disease. Int J Mol Sci. 2012;13(10):13501-20.

6. Zhao F, Guochun L, Yang Y, et al. A network pharmacology approach to determine active ingredients and rationality of herb combinations of modified-Simiaowan for treatment of gout. J Ethnopharmacol. 2015;168:1-16.

7. Yu G, Wang W, Wang X, et al. Network pharmacology-based strategy to investigate pharmacological mechanisms of Zuojinwan for treatment of gastritis. BMC Complementary Altern Med. 2018;18(1):292.

8. Tao WY, Xu X, Wang X, et al. Network pharmacology-based prediction of the active ingredients and potential targets of Chinese herbal Radix Curcumae formula for application to cardiovascular disease. Journal of Ethnopharmacol. 2013;145:1-10.

9. Cai BC, Wang TS, Krokawa M, et al. Cytotoxicities of alkaloids from processed and unprocessed seeds of Strychnos nux-vomica. Zhong Guo Yao Li Xue Bao. 1998;19(5):425-8. 
10. Deng XK, Yin W, Li WD, et al. The anti-tumor effects of alkaloids from the seeds of Strychnos nuxvomica on HepG2 cells and its possible mechanism. Journal of Ethnopharmacol. 2006;106(2):17986.

11. Yin W, Deng XK, Yin FZ, et al. The cytotoxicity induced by Strychnos nuxvomica proceeds via apoptosis by Cox 2 and caspase-3 in SMMC 7221 cells. Food Chem Toxicol. 2007;45(9):1700-8.

12. Shu GW, Mi X, Cai J,et al. Brucine, an Alkaloid From Seeds of Strychnos Nux-Vomica Linn., Represses Hepatocellular Carcinoma Cell Migration and Metastasis: The Role of Hypoxia Inducible Factor 1 Pathway. Toxicol Lett. 2018;222(2):91-101.

13. Rao PS, Ramanadham M, Prasad MNV. Antiproliferative and cytotoxic effects of Strychnos nuxvomica rootextract on the human multiple myeloma cell line-RPMI 8226. Food Chem Toxicol. 2009;47(2):283-8.

14. Rao PS, Prasad MNV. Strychnos nux-vomica Root Extract Induces Apoptosis in the Human Multiple Myeloma Cell Line-U266B1.Cell Biochem Biophys. 2013;66(3):443-450.

15. Zhao F, Guochun L, Yang Y, Shi L, Xu L, Yin L. A network pharmacology approach to determine active ingredients and rationality of herb combinations of modified-Simiaowan for treatment of gout. $J$ Ethnopharmacol. 2015;168:1-16.

16. Lyu M, Yan CL, Liu HX, Wang TY, Shi XH, Liu JP, et al. Network pharmacology exploration reveals endothelial inflammation as a common mechanism for stroke and coronary artery disease treatment of Danhong injection. Sci Rep. 2017;7(1):15427.

17. Fang HY, Zeng HW, Lin LM, Chen X, Shen XN, Fu P, et al. A network-based method for mechanistic investigation of Shexiang Baoxin Pill's treatment of cardiovascular diseases. Sci Rep. 2017;7:43632.

18. Yu GH, Wang WB, Wang X,et al. Network pharmacology-based strategy to investigate pharmacological mechanisms of Zuojinwan for treatment of gastritis.BMC Complement. Altern Med. 2018;18:292.

19. He D, Huang JH, Zhang ZY,et al. A Network Pharmacology-Based Strategy For Predicting Active Ingredients And Potential Targets Of LiuWei DiHuang Pill In Treating Type 2. Diabetes MellitusDrug Des Devel Ther. 2019;13:3989-4005.

20. Ru J, Li P, Wang J, Zhou W, Li B, et al. TCMSP: a database of systems pharmacology for drug discovery from herbal medicines. J cheminform. 2014;6:13.

21. Huang J, Cheung F, Tan HY, et al. Identification of the active compounds and significant pathways of yinchenhao decoction based on network pharmacology. Mol Med Rep. 2017;16(4):4583-92.

22. Huang J, Cheng F, Tan HY, et al. Identification of the active compounds and significant pathways of Yinchenhao decoction based on network pharmacology. Mol Med Rep. 2017;16(4):4583-92.

23. Stelzer G, Rosen N, Plaschkes I, et al. The GeneCards suite: from gene data mining to disease genome sequence analyses: the GeneCards suite. Cur Protoc Bioinf. 2016. doi:10.1002/cpbi.5.

24. Wang X, Shen Y, Wang S, et al. PharmMapper 2017 update: a web server for potential drug target identification with a comprehensive target pharmacophore database. Nucleic Acids Res. 2017;45:356-60. 
25. Breuza L, Poux S, Estreicher A, et al. The UniProtKB guide to the human proteome. Database Oxfo. 2016. doi:10.1093/database/bav120. Print 2016.

26. Szklarczyk D, Morris JH, Cook H, et al. The STRING database in 2017: quality-controlled proteinprotein association networks, made broadly accessible. Nucleic Acids Res. 2017;45(1):362-8.

27. Shannon P, Markiel A, Ozier O, et al. Cytoscape: a software environment for integrated models of biomolecular interaction networks. Genome Res. 2003;13:2498-504.

28. Ba Q, Li J, Huang C, et al. Topological, functional, and dynamic properties of the protein interaction networks rewired by benzo(a)pyrene. Toxicol Appl Pharmacol. 2015;283(2):83-91.

29. Guo R, Zhang X, Su J, et al. Identifying potential quality markers of Xin-Su-Ning capsules acting on arrhythmia by integrating UHPLC-LTQ-Orbitrap, ADME prediction and network target analysis. Phytomedicine. 2018;44:117-28.

30. Zhu JF, Yi XJ, Zhang YW, et al. Systems pharmacology-based approach to comparatively study the independent and synergistic mechanisms of danhong injection and naoxintong capsule in ischemic stroke treatment. Evid Based Complement Alternat Med. 2019; 1-17.

31. Zhong JL, Liu ZH, Zhou XX, Xu J. Synergic anti-pruritus mechanisms of action for the radix sophorae flavescentis and fructus. Cnidii herbal pairMolecules. 2017;22(9):1465.

32. Yue SJ, Xin LT, Fan YC, Li SJ, Tang YP, et al. Herb pair Danggui-Honghua: mechanisms underlying blood stasis syndrome by system pharmacology approach. Sci rep. 2017;7:40318.

33. Ramakrishnan V, Kumar S. PI3K/AKT/mTOR pathway in multiple myeloma: from basic biology to clinical promise. Leuk Lymphoma. 2018;59(11):2524-34.

34. Chen $\mathrm{Y}$, Huang R, Ding JH, et al. Multiple myeloma acquires resistance to EGFR inhibitor via induction of pentose phosphate pathway. Sci Rep. 2015;5:9925.

35. Zuehlke AD, Beebe K, Neckers L, Prince T, et al. Regulation and function of the human HSP90AA1 gene.Gene.2015;570(1): 8-16.

36. Wang X, Yan Z, Fulciniti M,et al. Transcription factor-pathway coexpression analysis reveals cooperation between SP1 and ESR1 on dysregulating cell cycle arrest in non-hyperdiploid. multiple myelomaLeu. 2014;28(4):894-903.

37. He J, Liu Z, heng Y, et al. P38 MAPK in myeloma cells regulates osteoclast and osteoblast activity and induces bone destruction. Cancer Res. 2012;72(24):6393-402.

38. Xiao W, Xu Z, Chang S,et al. Rafoxanide, an organohalogen drug, triggers apoptosis and cell cycle arrest in multiple myeloma by enhancing DNA damage responses and suppressing the p38 MAPK. Cancer Lett. 2019;444:45-59.

39. Podar K, Anderson KC. The pathophysiologic role of VEGF in hematologic malignancies: therapeutic implications. Blood. 2005;105:1383-95.

40. Rao L, Veirman KD, Giannico D,et al.Targeting angiogenesis in multiple myeloma by the VEGF and HGF blocking DARPin ${ }^{\circledR}$ protein MP0250: a preclinical study.Oncotarget. 2018;9(17):13366-13381. 
41. Mayer IA, Arteaga CL. The PI3K/AKT pathway as a target for cancer treatment. Annu Rev Med. 2016;67:11-28.

42. Fruman DA, Rommel C. PI3K and cancer: lessons, challenges and opportunities. Nat Rev Drug Discov. 2014;13(2):140-56.

43. Vivanco I, Sawyers CL. The phosphatidylinositol 3-kinase AKT pathway in human cancer. Nat Rev Cancer. 2002;2(7):489-501.

44. Yang J, He J, Wang J, Cao Y, Ling J, Qian J, et al. Constitutive activation of p38 MAPK in tumor cells contributes to osteolytic bone lesions in multiple myeloma. Leu. 2012;26(9):2114-23.

45. Uchiyama H, Barut BA, Mohrbacher AF, Chauhan D, Anderson KC. Adhesion of human myelomaderived cell lines to bone marrow stromal cells stimulates interleukin-6 secretion. Blood. 1993;82:3712-20.

46. Vacca A, Ribatti D, Roncali L, Ranieri G, Serio G, Silvestris F, et al. Bone marrow angiogenesis and progression in multiple myeloma. Br J Haematol. 1994;87(3):503-8.

47. Wilhelm SM, Carter C, Tang L, Wilkie D, McNabola A, Rong H, et al. BAY 43-9006 exhibits broad spectrum oral antitumor activity and targets the RAF/MEK/ERK pathway and receptor tyrosine kinases involved in tumor progression and angiogenesis. Cancer Res. 2004;64(19):7099-109.

48. Mahtouk K, Cremer FW, Rème T,et al. Heparan sulphate proteoglycans are essential for the myeloma cell growth activity of EGF-family ligands in multiple myeloma.Oncogene. 2006;25(54):7180-7191.

\section{Figures}




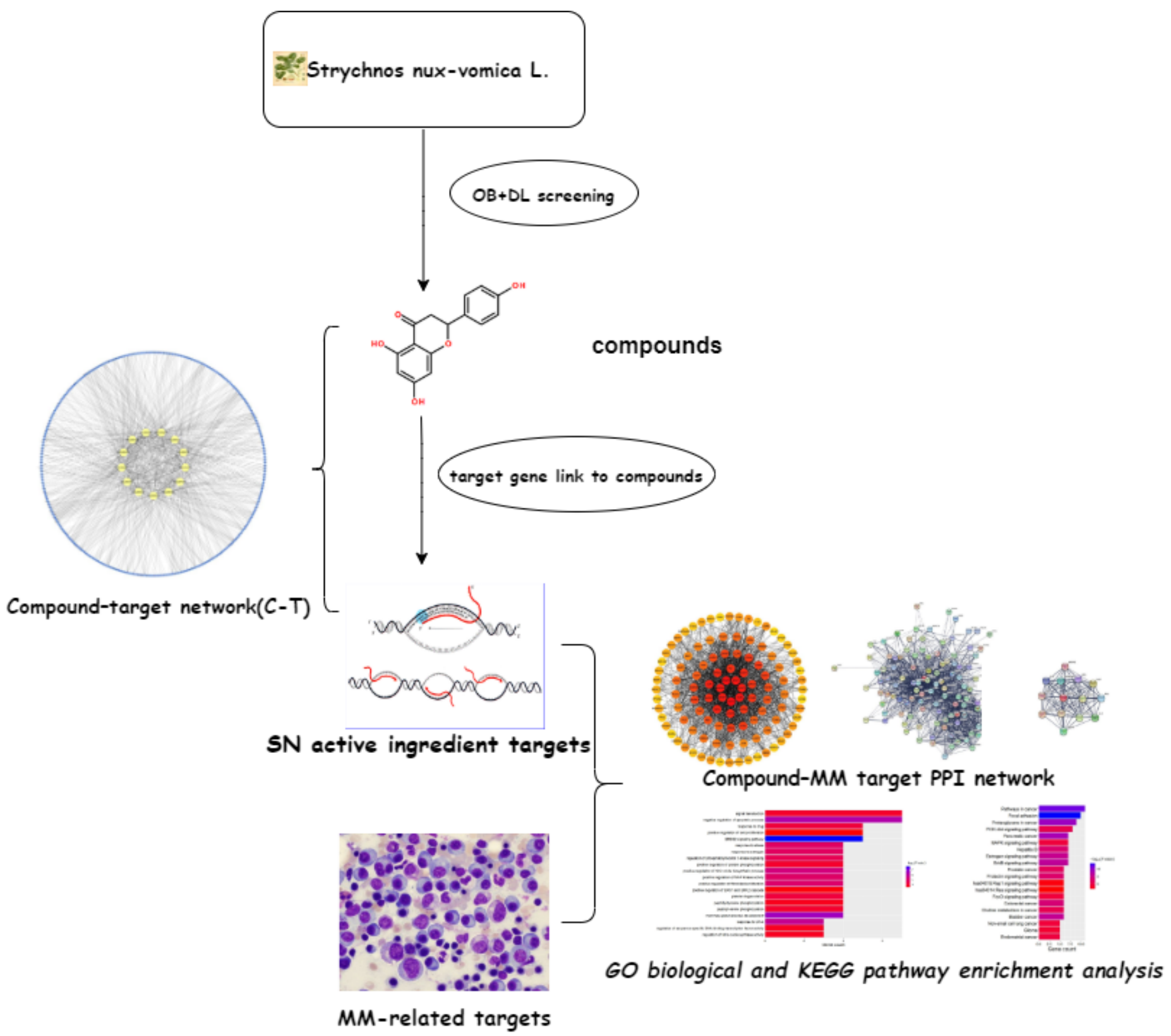

Figure 1

Workflow of network pharmacology analysis. 


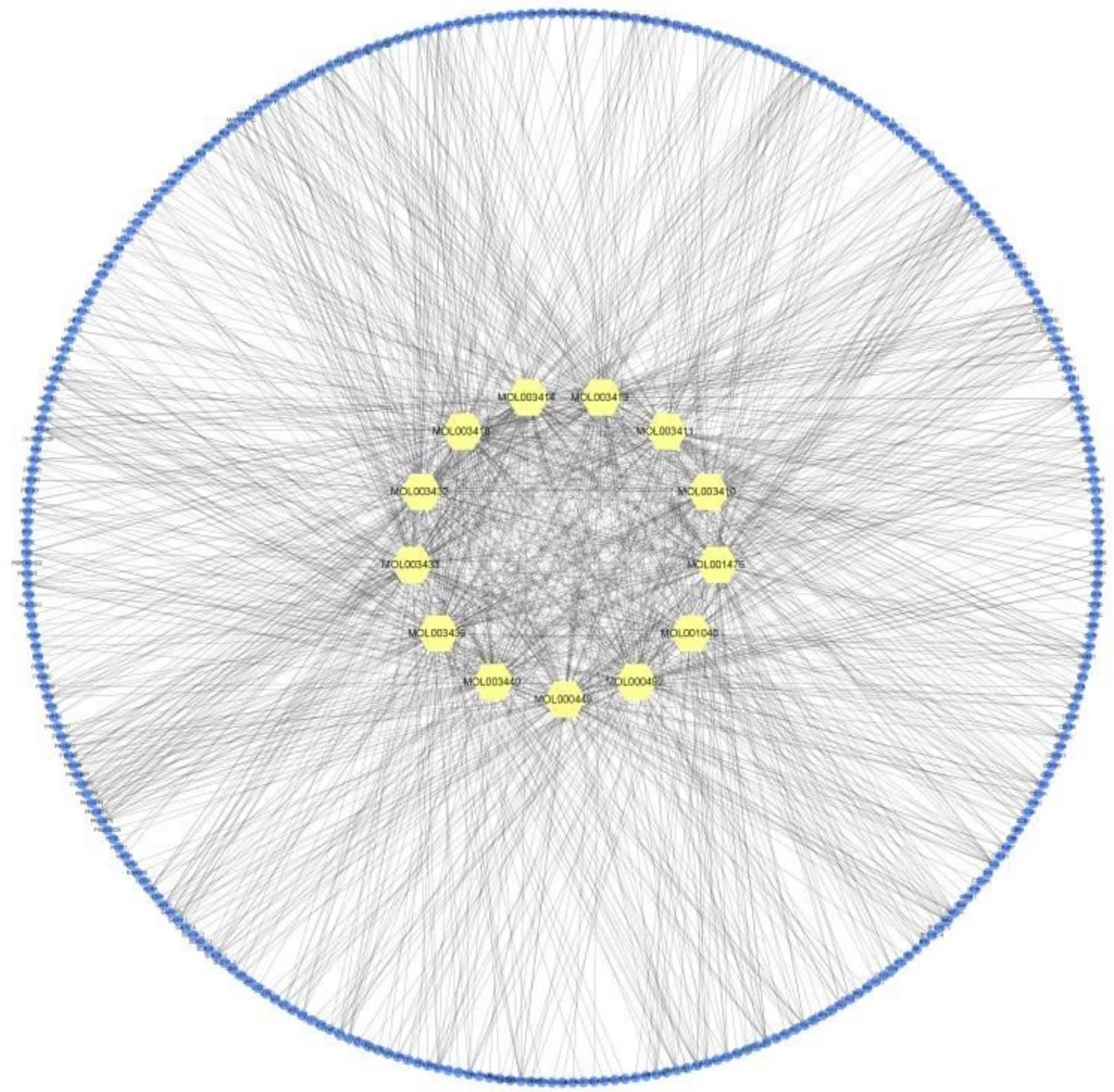

Figure 2

Compound-target network(C-T) of Strychnos nux-vomica L. The C-T network that consists of 221 nodes and 390 edges. Yellow and blue nodes denote the compounds and targets respectively. 


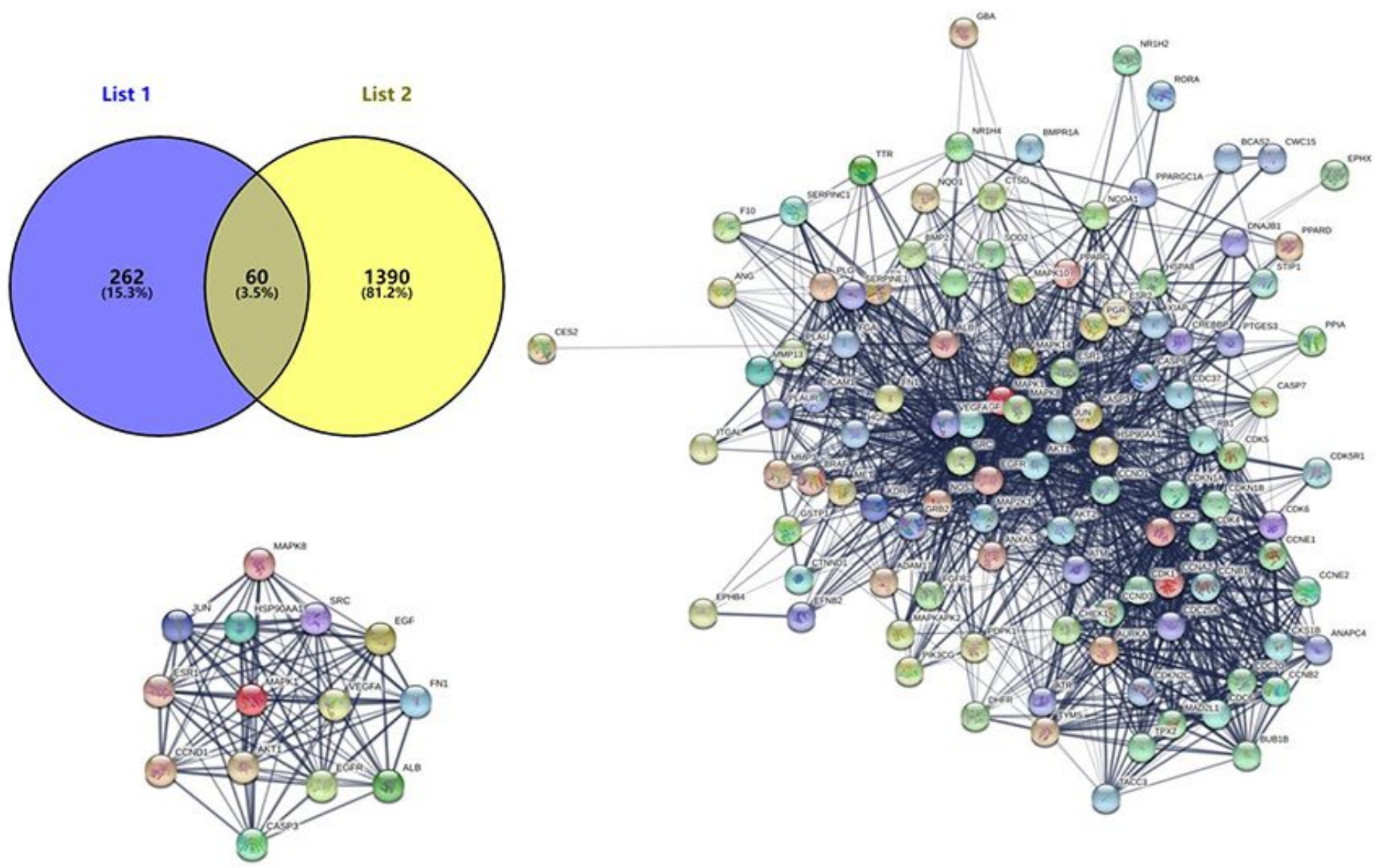

Figure 3

(a): 60 of the identified potential targets of the SN were also Multiple Myeloma- related targets by the Venn diagram.(b): Compound -Multiple Myeloma PPI network.(c):PPI network of the 14 hub tagets consisted of 14 nodes and 91 edges. 


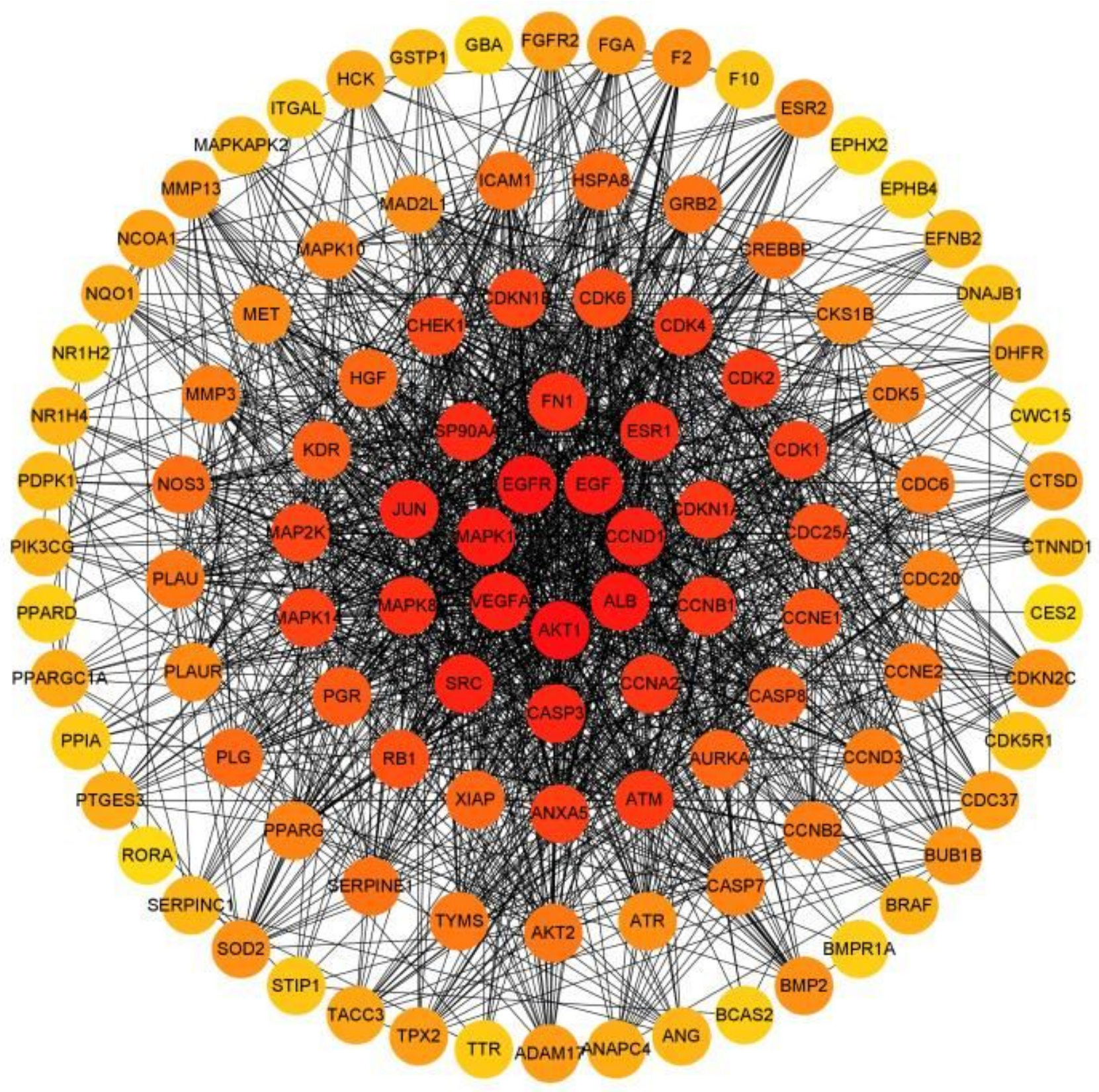

Figure 4

Construction of Compound-Multiple Myeloma network.the node with different depth of colour represented the different degree of nodes. 
signal transduction

negative regulation of apoptotic process

response to drug positive regulation of cell proliferation

ERBB2 signaling pathway

response to stress

response to estrogen

regulation of phosphatidylinositol 3-kinase signaling

positive regulation of protein phosphorylation

positive regulation of nitric oxide biosynthetic process

positive regulation of MAP kinase activity

positive regulation of fibroblast proliferation

positive regulation of ERK1 and ERK2 cascade

platelet degranulation

peptidyl-tyrosine phosphorylation

peptidyl-serine phosphorylation

mammary gland alveolus development

response to UV-A

regulation of sequence-specific DNA binding transcription factor activity

regulation of nitric-oxide synthase activity

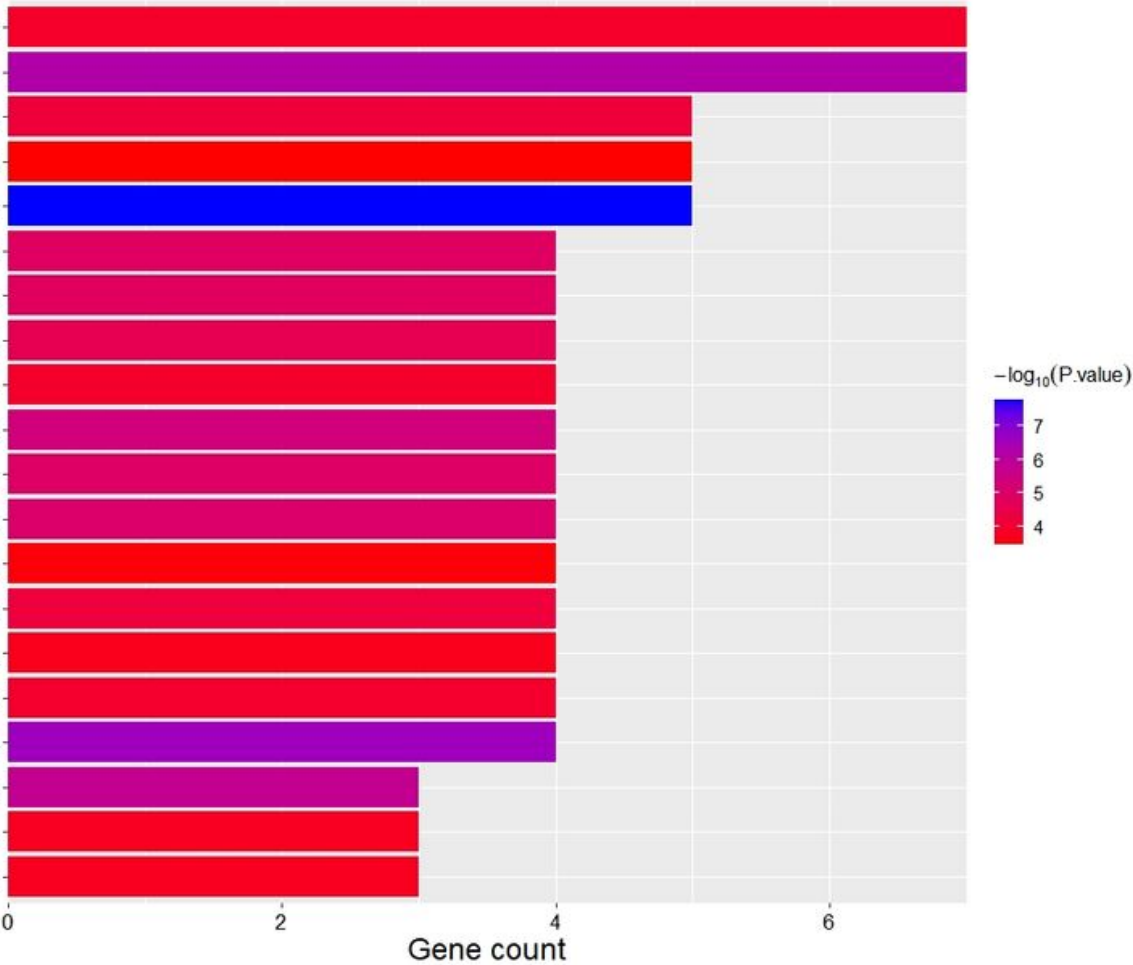

\section{Figure 5}

GO biological process by major hubs from the DAVID database. 


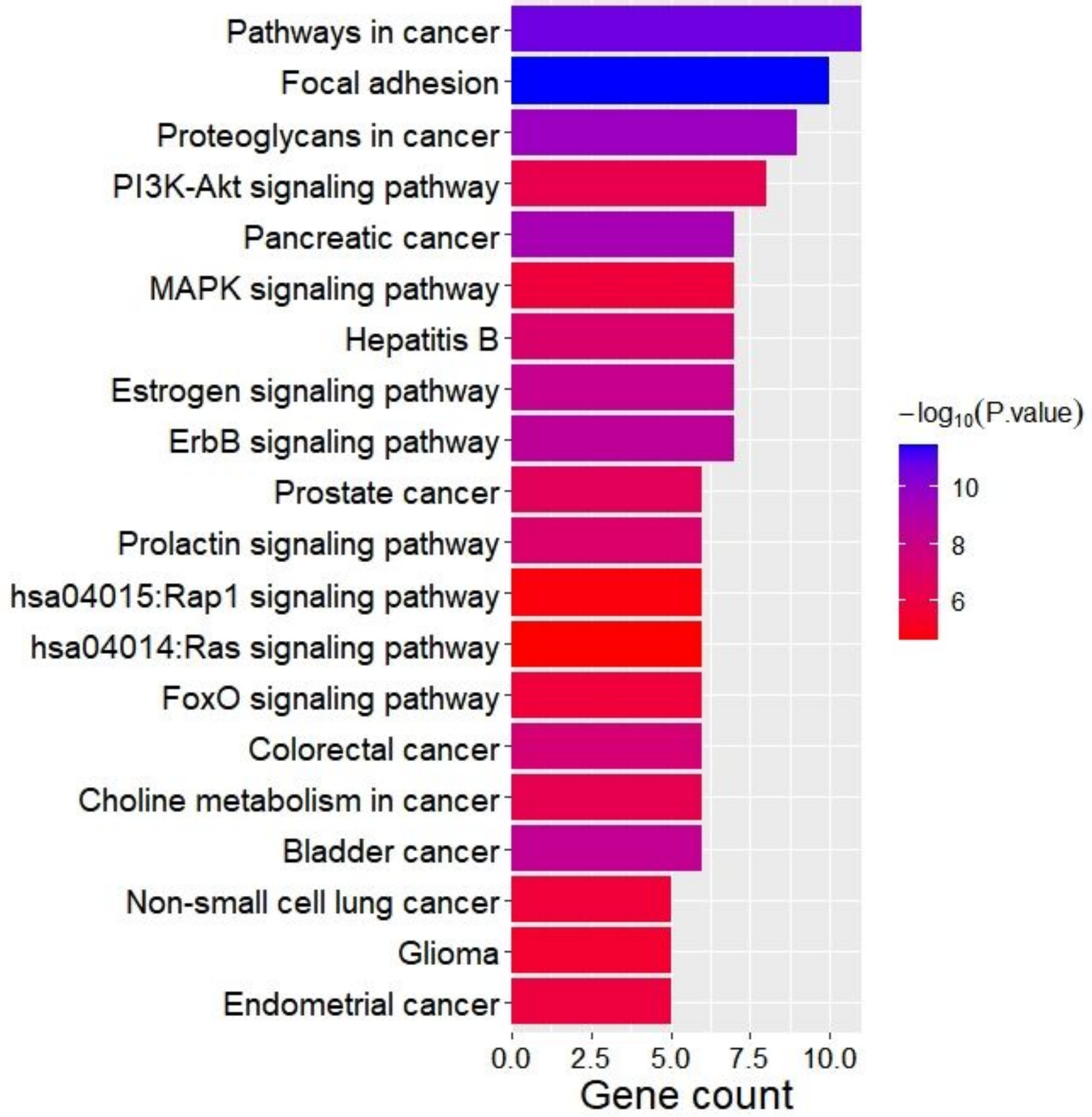

Figure 6

main pathways enriched by major hubs from the DAVID database. 